\title{
Methodology for preparing coal seams with high methane content by complex impact method
}

\author{
Dung Tien Thai $V u^{1}{ }^{*}$, Thanh Van Tran ${ }^{2}$, Mien Van Nguyen ${ }^{3}$, Tinh Van Do 4 \\ ${ }^{1}$ Hanoi University of Mining and Geology, Hanoi, Vietnam \\ 2 Vietnam Mining Science and Technology Asscociation, Hanoi, Vietnam \\ ${ }^{3}$ Dong Bac Corporation - Ministry of National Defence, Quang Ninh, Vietnam \\ ${ }_{4}^{4}$ Vietnam National Coal - Mineral Industries Holding Corporation Limited, Hanoi, Vietnam
}

ARTICLE INFO ABSTRACT

Article history:

Received 12th Apr. 2021

Revised 10th July 2021

Accepted 08 ${ }^{\text {th }}$ Aug. 2021

Keywords:

Gas emission,

High methane content, Impact vibration,

Low permeability, Preparation of coal seams.
Safety is always a top priority in underground coal mining, especially when mining at great depths, which is accompanied by an increase in methane content of the coal seams. The high concentration of methane in mine airstream is cause of many negative effects on various underground works. In some cases, when the methane content in underground areas reaches the limit value, mine fires can occur with great loss of people and equipment. In many countries, where the coal mining industry is developed, practical experience in deep mining has shown thatcurrent popular methods of coal seams preparation can not always bring absolute safety in working faces, especially when extracting and recovering coal seams with high methane content. The purpose of this study is to develop an appropriate coal seam preparation method that can minimize the risk of methane emissions, which is one of the causes of unsafety in the underground coal mining. In this paper, the authors propose to use a combination of different impact methods through boreholes to control the intensity of methane release from high methane content coal seams. The combined use of hydraulic and vibration methods will greatly increase the network of man-made cracks in the coal mass, which allows methane to escape more easily and reduce the gas content of coal block before extracting. The research and testing of the complex impact method in real conditions has yielded positive results: the ability to release gas increased by at least $3 \div 5$ times and the degassing efficiency reached over 60\% for coal seams, which has low permeability. After research and analysis, the authors conclude that the complex effect method is a promising new direction to support the control of methane emissions and contribute to the improvement of technical - safety effects when extracting coal seams with high methane content.

Copyright (C) 2021 Hanoi University of Mining and Geology. All rights reserved.

${ }^{*}$ Corresponding author

E - mail: vuthaitiendung@humg.edu.vn

DOI: $10.46326 / J M E S .2021 .62(5 a) .09$ 


\title{
Tạp chí Khoa học Kỹ thuật Mỏ - Địa chất
}

Trang điện tư: http://tapchi.humg.edu.vn

\section{Phương pháp luận về chuẩn bị ruộng mỏ bằng phương pháp tác động phức hợp đối với vỉa than có độ chứa khí mêtan cao}

\author{
Vũ Thái Tiến Dũng 1,*, Trần Văn Thanh ${ }^{2}$, Nguyễn Văn Miền ${ }^{3}$, Đỗ Văn Tỉnh 4 \\ 1 Trường Đại học Mỏ-Địa chất, Hà Nội, Việt Nam \\ ${ }^{2}$ Hội Khoa học và Công nghệ Mỏ Việt Nam, Hà Nội, Việt Nam \\ 3 Tổng Công ty Đông Bắc, QuangNinh, Việt Nam \\ ${ }^{4}$ Tập đoàn Công nghiệp Than - Khoáng sản Việt Nam, Hà Nội, Việt Nam
}

THÔNG TIN BÀI BÁO

TÓM TẮT

Quá trình:

Nhận bài 12/4/2021

Sứa xong 10/7/2021

Chấp nhận đăng 08/8/2021

Tù̀ khóa:

Chuẩn bị vỉa than,

Độ chứa khí mêtan cao,

Độ thấm thấp,

Phát thải khí,

Tác động rung động.
An toàn luôn là vấn đề quan trọng trong khai thác than hầm lò, đặc biệt khi khai thác xuống sâu cùng với sự gia tăng độ chứa khí mêtan của các vỉa than. Hàm lượng khí mêtan tăng cao trong luồng không khí mỏ gây ra nhiều ảnh hưởng tiêu cực đối với các công tác sản xuất, trong một số trường hợp có thể gây cháy mỏ với thiệt hại lớn về con người và thiết bị. Mục đích của nghiên cúvu nhằm xây dựng phương pháp chuẩn bị ruộng mỏ thích hợp để giảm thiểu nguy cơ phát thải khí mêtan gây mất an toàn trong khu vực khai thác. Bài báo đề xuất sử dung kết hợp các phương pháp tác động khác nhau thông qua các lỗ khoan nhằm kiểm soát khả năng giải phóng khí mêtan tù̀ vỉa. Việc sử dụng kết hợp phương pháp tác động thủy lực và tác động rung động sẽ làm gia tăng đáng kể mạng lưới các vết nứt nhân tạo trong khối than, tạo điều kiện cho khí mêtan có thể thoát ra dễ dàng, làm giảm hàm lượng chứa khí của khối than trước khai thác. Nghiên cứu và thử nghiệm phương pháp trong điều kiên via thực tế mang lai nhũng kết quả khả quan: khả năng thoát khí tăng tối thiểu $3 \div 5$ lần và hiệu suất khử khí đạt trên $60 \%$ đối vói các vỉa than có độ thấm thấp. Như vậy, phương pháp tác động phức hợp là một hướng đi mới có triển vọng nhằm hỗ trợ công tác kiểm soát sự phát thải khí mêtan, góp phần nâng cao hiệu quả kỹ thuật - an toàn khi khai thác các vỉa than có độ chứa khí mêtan cao.

C 2021 Trường Đại học Mỏ - Địa chất. Tất cả các quyền được bảo đảm.

\section{Mở đầu}

Công nghệ khai thác lò chợ là nền tảng cơ bản của công tác sản xuất mỏ cần được cải tiến không ngừng. Để nâng cao hiệu quả khai thác lò chợ,

*Tác giả liên hệ

E - mail: vuthaitiendung@humg.edu.vn DOI: 10.46326/JMES.2021.62(5a).09 phương pháp tích hợp mới đã được đề xuất nhằm khử khí vỉa than, giúp thu hồi khí mêtan tối đa. Khi công tác mỏ diễn ra ở độ sâu tương đối lớn $(1.000 \div 15.00 \mathrm{~m})$, năng suất lò chợ giảm đáng kể do lượng lớn khí mêtan thải ra từ gương lò chợ và các gương lò chuẩn bị (Pavlenko, 2018). Hiện nay, để giải quyết vấn đề gia tăng công suất lò chợ, tức là tăng khả năng sinh lời của mỏ do tăng cường độ khai thác than ở các vỉa chứa khí mêtan, cần giải quyết vấn đề đảm bảo an toàn khai thác khi chịu 
ảnh hưởng của khí mêtan. Nghĩa là, để có thể gia tăng cường độ khai thác lò chợ với các tổ hợp thiết bị cơ giới hóa hiệu suất cao, cần đảm bảo loại bỏ rào cản về sự nguy hiểm của khí mêtan ở gương lò chợ.

Ở nước ta, các phương pháp chuẩn bị vỉa than hiện đang áp dụng về cơ bản vẫn đảm bảo an toàn và mang lại hiệu quả trong quá trình sản xuất. Tuy nhiên, khi khai thác các vỉa than có độ chứa khí mêtan cao, đặc biệt là đối với những mỏ loại III hoặc siêu hạng với độ chứa khí $\geq 4,5 \mathrm{~m}^{3} / \mathrm{TKC}$, thực tế từ các nước có ngành công nghiệp khai thác than phát triển với các mỏ có độ sâu khai thác lớn đã chỉ ra rằng các phương pháp chuẩn bị vỉa than phổ biến hiện nay không phải lúc nào cũng có thể mang lại sự an toàn tuyệt đối cho công tác khai thác ở gương lò chợ. Việc lựa chọn phương pháp chuẩn bị hợp lý đối với các vỉa than có độ chứa khí, xuất khí mêtan cao cần được đánh giá dựa trên việc đảm bảo hàm lượng khí mêtan trong các đường lò chuẩn bị phải được giảm thiểu để đạt mức tối thiểu cho phép, tạo điều kiện cho các công tác mỏ khác được diễn ra trong môi trường an toàn, thuận lợi.

\section{Xây dựng cơ sở lý thuyết của phương pháp tác động phức hợp}

Khi khai thác các vỉa than có độ chứa khí mêtan cao, việc lựa chọn công nghệ khai thác, tổ hợp thiết bị sản xuất bắt buộc phải dựa trên mục tiêu tăng năng suất lò chọ̣ cùng với giảm "rào cản khí". Nghĩa là phương án được lựa chọn phải đảm bảo khắc phục, loại trừ tối đa ảnh hưởng của khí mêtan đến việc gia tăng sản lượng lò chợ.

Trong trường hợp này, để gia tăng sản lượng lò chợ cần thực hiện hai nhiệm vụ cơ bản sau:

- Giảm nồng độ khí mêtan trong luồng gió thải từ lò chợ xuống dưới giá trị tối đa cho phép theo Quy chuẩn kỹ thuật quốc gia về an toàn trong khai thác than hầm lò (Bộ Công thương, 2011);

- Giảm nồng độ khí mêtan trong các gương lò chuẩn bị.

Để giải quyết các vấn đề trên, các tác giả đề xuất sử dụng phương pháp kết hợp giữa tác động rung động trên khối than chứa khí nhằm giảm sự liên kết, gia tăng vết nứt trên khối than để tăng cường giải phóng khí và sau đó loại bỏ mêtan bằng phương pháp khử khí để giảm nồng độ của khí mêtan trong môi trường khí của các đường lò. Tác động này kết hợp với phương pháp phá vỡ thủy lực cho phép tăng năng suất lò chợ xét theo phương diện ảnh hưởng bởi các yếu tố khí và đảm bảo an toàn cho hoạt động khai thác (Jia và nnk., 2012; Li và nnk., 2016; Wei và nnk., 2016; Yang, 2014; Yao và nnk., 2016).

Đề xuất này đã dẫn đến nhận thức rằng tác động rung động là một yếu tố bắt buộc trong phương pháp phức hợp để có được khả năng nứt nẻ và tạo ra dòng chảy lọc khí trong các khối than nguyên của vỉa than (Korshunov và nnk., 2014; Pavlenko, 2018; Slastunov và nnk., 2018; Yang, 2014).

Hình 1 mô tả vùng ảnh hưởng của phương pháp tác động phức hợp lên vỉa than khi chuẩn bị vỉa theo phương pháp chia cột với việc áp dụng tác động thủy lực nhờ việc bơm nước vào thông qua các lỗ khoan từ mặt đất ở giai đoạn đầu kết hợp với tác động rung động trong giai đoạn sau đó.

Dựa theo kết quả của một số công trình nghiên cứu thực tế tại các mỏ than ở nước ngoài, với độ cứng thông thường của than $f=2 \div 3$ thì tác động thủy lực nên được thiết lập với thông số áp suất $P$ $=25 \div 23 \mathrm{MPa}$ và lưu lượng phun $q=25 \div 45$ lít/giây. Đây là thông số được xác định ở phần lỗ khoan có tiếp xúc trực tiếp tới vỉa than, các thông số đầu vào cần phải tính toán đến các yếu tố liên quan đến công tác đưa nước vào vỉa thông qua các các lỗ khoan từ bề mặt. Trong trường hợp này, vùng ảnh hưởng của tác động thủy lực thường có dạng elip được phát triển theo hướng của hệ thống vết nứt chính với bán kính trục chính và trục phụ trong khoảng $r=70 \div 140 \mathrm{~m}$.

Trong quá trình chuẩn bị vỉa than có độ chứa khí mêtan cao, việc áp dụng phương pháp tác động phức hợp như trên hứa hẹn mang lại những kết quả tích cực nhằm góp phần kiểm soát hàm lượng khí mêtan còn lại của khối than và hàm lượng khí mêtan thoát ra không gian làm việc (Korshunov và nnk., 2014; Pavlenko và nnk., 2015). Để nâng cao chất lượng của công tác chuẩn bị, các lỗ khoan sử dụng cần phải được tính toán đến vị trí thi công để phù hợp với vị trí đào các đường lò chuẩn bị.

Một vài công trình nghiên cưu đã chỉ ra rằng, phương pháp tác động rung động cho phép tăng khả năng thoát khí gấp $3 \div 5$ lần và hệ số hiệu suất khử khí tăng lên $60 \div 70 \%$ trong điều kiện khai thác khối than có độ thấm thấp (Panyshko và nnk., 1999; Pavlenko và Barnov, 2019; Pavlenko và nnk., 2015). 


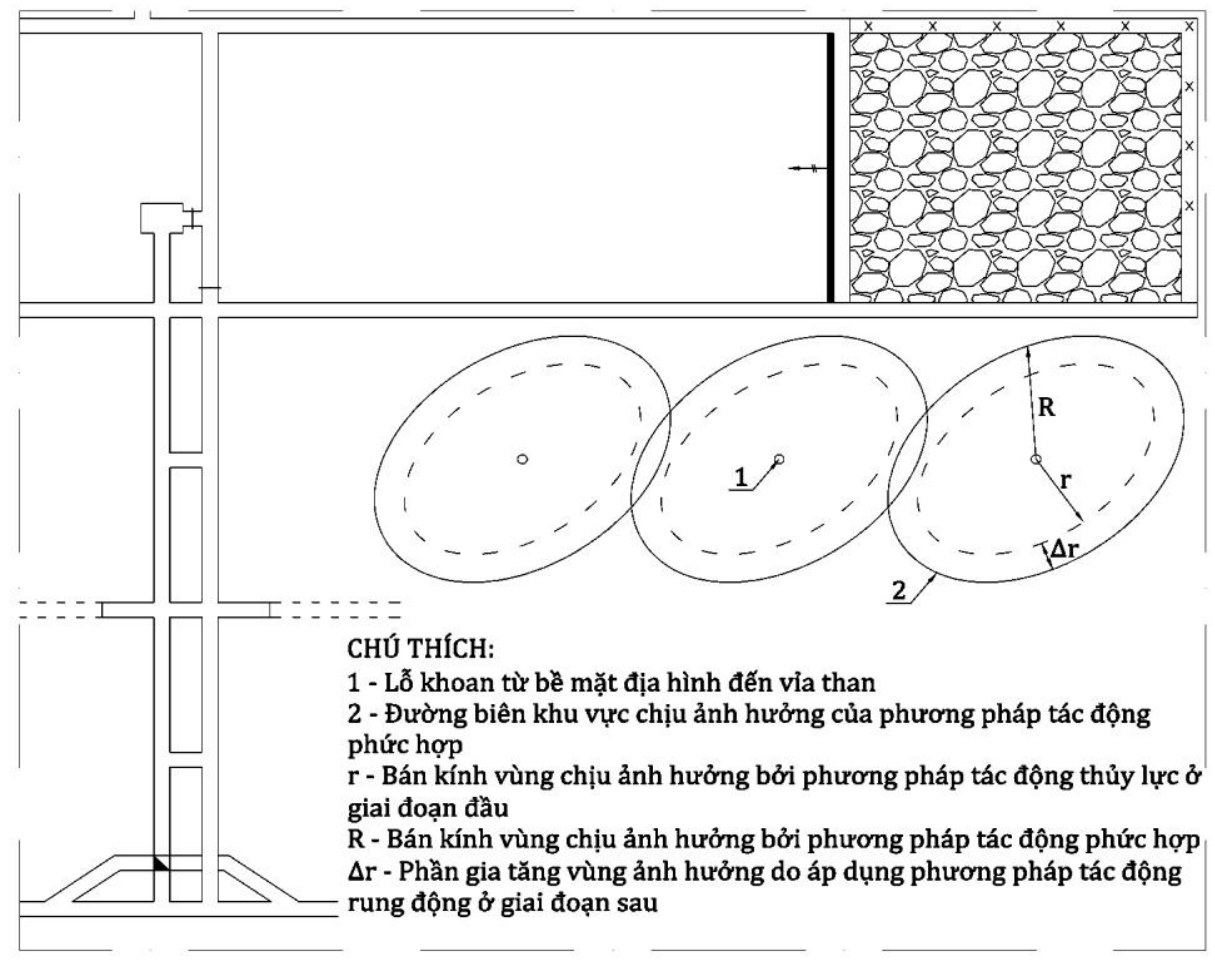

Hình 1. Sơ đồ mô tả vùng ảnh hưởng của phương pháp tác động phức hợp (Pavlenko và nnk., 2020).

\section{3. Áp dụng phương pháp phức hợp trong quá trình chuẩn bị vỉa than có hàm lượng khí mêtan cao}

Một sơ đồ kết hợp nhằm giảm độ chứa khí mêtan trong vỉa than cho các vỉa mỏng và dày trung bình bằng việc sử dụng các "lỗ khoan rung động" đã được phát triển (Hình 2). Quá trình khử khí của vỉa than nên được thực hiện ở chế độ phức hợp, gồm phân tách nhờ thủy lực và tác động rung. Để đáp ứng nhu cầu gia tăng công suất của mỏ, công tác khấu tại lò chợ cần được diễn ra liên tục ở cường độ cao sẽ làm gia tăng lượng khí mêtan thoát vào không gian lò chợ. Nồng độ khí mêtan trong không khí mỏ tăng lên làm giảm độ an toàn của các hoạt động khai thác mỏ.

Trong sơ đồ công nghệ được thể hiện trên Hình 2 các lỗ khoan tác động thủy lực 1 được khoan từ mặt đất đến khối than chuẩn bị khai thác với khoảng cách giữa các lỗ khoan $b=50 \div 70 \mathrm{~m}$ nhằm mục đích tháo khí có áp sơ bộ để khí tự thoát ra từ vỉa qua lỗ khoan. Tiếp đó, trước khi lò chợ khai thác đến khối than đang chuẩn bị, từ các đường lò chuẩn bị cần thực hiện khoan các lỗ khoan tác động rung động 2 có chiều dài $L_{1}=100 \div 120 \mathrm{~m}$ với khoảng cách giữa các lỗ dao động trong khoảng $a$ $=10 \div 15 \mathrm{~m}$ (Pavlenko và nnk., 2020). Sự kết hợp giữa 2 hệ thống lỗ khoan trên sẽ nâng cao hiệu quả thoát khí mêtan cho các vỉa than chứa khí, giải phóng một lượng đáng kể khí mêtan nhằm đảm bảo an toàn cho công tác khai thác mỏ.

Sơ đồ công nghệ phức hợp để chuẩn bị cho các vỉa than chứa khí trong trường hợp khái quát nhất được xây dựng dựa trên các thông số rung động khuyến nghị, các thông số này được xác định với mục tiêu cuối cùng là đảm bảo sự an toàn và hiệu quả của hoạt động khai thác. Do đó, theo quan điểm chung, việc thay đổi trị số các yếu tố rung động riêng lẻ trong mối quan hệ giữa không gian và thời gian tác động lên khối than sẽ tạo ra các sơ đồ công nghệ phức hợp khác nhau. Việc sử dụng hiệu ứng rung có mức tiêu thụ năng lượng thấp cho phép loại bỏ "rào cản khí", nâng cao năng suất lò chợ và giảm thiểu tối đa chi phí thông gió liên quan đến gia tăng nồng độ khí mêtan. Việc sử dụng hiệu ứng rung động cũng được coi như một phương pháp phụ trợ nhằm khử khí trong không gian đã khai thác, giảm nồng độ khí mêtan thoát ra khu vực làm việc của lò chợ.

Trong quá trình khai thác, mỗi vỉa than sẽ có độ 


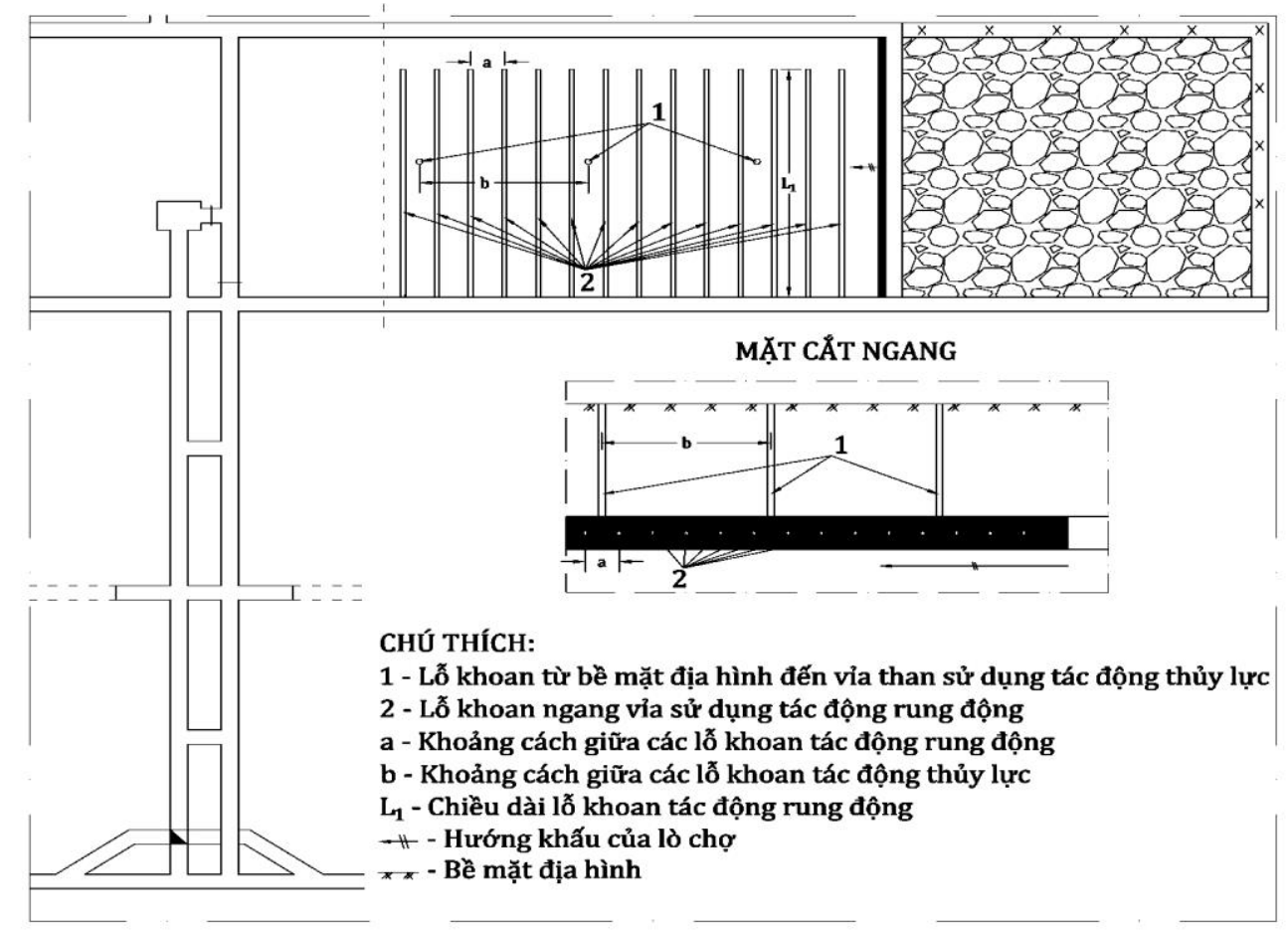

Hình 2. Sơ đồ công nghệ chuẩn bị vỉa than có độ chứa khí mêtan cao bằng phương pháp tác động phức hợp (Pavlenko và nnk., 2020).

thoát khí khác nhau, điều này được quyết định bởi phương thức khai thác và độ chứa khí của khối than nguyên ban đầu. Vì vậy, việc xây dựng, tính toán áp dụng các phương pháp tác động trong quá trình chuẩn bị vỉa cần phải dựa trên các thông số của vỉa than trong điều kiện thực tế.

Tác động rung được thực hiện bằng nhiều phương pháp khác nhau, bao gồm cả phương pháp sử dụng rung chấn cơ học tạo ra từ các thiết bị hỗ trợ, có thể cung cấp sự nứt nẻ đáng kể lên khối than. Kết hợp với tác động thủy lực trước đó, tác động rung sẽ tạo ra hệ thống các đứt gãy bổ sung làm tăng bán kính của khu vực chịu ảnh hưởng của tác động phức hợp (Korshunov và nnk., 2014). Khi thiết kế các biện pháp tác động phức hợp đối với các vỉa than chứa khí cần phải gắn chặt chúng với các giải pháp công nghệ áp dụng và bản chất của các hoạt động khai thác như công việc chuẩn bị và khai thác, công tác khử khí và các công việc phụ trợ khác.

\section{Kết luận}

Đã có những nghiên cứu của nhiều tác giả khác nhau về phương pháp tháo khí mêtan trong quá trình chuẩn bị các vỉa than có độ chứa khí mêtan cao, những nghiên cứu này về cơ bản chỉ đưa ra những phương án kỹ thuật độc lập dựa trên cơ sở lý thuyết được xây dựng. Mặc dù chưa được áp dụng rộng rãi tại Việt Nam nhưng qua việc thử nghiệm tại nhiều mỏ than hầm lò trên thế giới cho thấy hiệu quả của từng phương án khi áp dụng riêng lẻ thường mang lại hiệu quả không cao.

Trong bài báo này, các tác giả đã đề xuất sử dụng phương pháp tác động phức hợp trong quá trình chuẩn bi vỉa than để tháo khí sớm cho vỉa có độ chứa khí mêtan cao. Sơ đồ công nghệ của phương pháp tác động phức hợp áp dụng cho các vỉa than có độ chứa khí mêtan cao được hiểu là tập hợp các quy trình và trình tự thực hiện, cho phép thực hiện các tác động khác nhau theo một trình tự nhất định, bổ sung cho nhau và phát triển chúng từ giai đoạn này sang giai đoạn khác, để thực hiện các công đoạn sản xuất chính và phụ trợ nhằm thực hiện quá trình khử khí. Bản chất của phương pháp là sử dụng kết hợp các hiệu ứng tác động khác nhau nhằm thay đổi theo hướng tích cực quá trình hình thành và phát triển các hệ thống nứt nẻ trong khối than trước khi đưa vào khai thác nhằm gia tăng sự thoát khí mêtan. Đây là một phương án ưu tiên trong việc chuẩn bị sớm khối than chứa khí để khai thác ở cường độ cao dựa trên việc tính 
đến các loại tác động tích cực và trình tự thực hiện chúng, trong đó thường sử dụng các lỗ khoan khử khí sớm bằng tác động thủy lực và bổ sung các tác động rung động trong giai đoạn sau.

Thông qua các lỗ khoan từ mặt đất và từ các công trình mỏ, việc sử dụng các tác động khác nhau nhằm tạo ra một mạng lưới các vết nứt nhân tạo phân nhánh đáng kể trong vỉa và trong vùng lân cận của vỉa bị ảnh hưởng bởi lỗ khoan làm tăng độ thoát khí mêtan trong giai đoạn chuẩn bị. Từ đó cung cấp điều kiện để thực hiện những biện pháp bổ sung nhằm hòa loãng nồng độ không khí mỏ về nồng độ cho phép trước khi đưa khối than vào khai thác, góp phần đảm bảo an toàn và nâng cao hiệu quả sản xuất.

\section{Đóng góp của các tác giả}

Trần Văn Thanh - xây dựng ý tưởng, bố cục bài báo, đọc bản thảo bài báo và cho ý kiến đóng góp; Nguyễn Văn Miền và Đỗ Văn Tỉnh - nghiên cứu thực trạng, thu thập số liệu. Vũ Thái Tiến Dũng xử lý số liệu và viết bản thảo.

\section{Tài liệu tham khảo}

Bộ Công thương, (2011). QCVN 01:2011/BCT Quy chuẩn kỹ thuật quốc gia về an toàn trong khai thác than hầm lò.

Jia, P., Tang, C.A., and Zhang, Y.B., (2012). Numerical stady on zonal disintegration of rock mass around deep underground openings. Harmonising Rock Engineering and the Environment: proceedings of the 12th ISRM International Congress on Rock Mechanics, Florida: CRC Press, 179-180.

Korshunov, G.I., Seregin, A.S., Sadov, A.P., and Komissarov, I.A., (2014). Degassing of coal seams based on cyclic hydrodynamic action (Дегазация угольных пластов на основе циклического гидродинамического воздействия). Mining information and analytical bulletin (Горный информационноаналитический бюллетень), 3, 29-35. (In Russian).

Li, X.L., Wang, E.Y., Li, Z.H., (2016). Rock burst monitoring by integrated microseismic and electromagnetic radiation methods. Rock Mechanics \& Rock Engineering, 49(11), 43934406.
Panyshko, A.Y., Rozhon, V.D., and Pavlenko, M.V., (1999). Investigation of the process of methane recovery from a coal mass under vibration (Исследование процесса метаноотдачи из угольного массива при вибровоздействии). Mining information and analytical bulletin (Горный информационноаналитический бюллетень), 8, 46-47. (In Russian).

Pavlenko, M.V., (2018). Justification of the technology for the preparation of a gasbearing coal seam based on a complex impact (Обоснование технологии подготовки газоносного угольного пласта на базе комплексного воздействия). Mining information and analytical bulletin (Горный информационно-

аналитическийбюллетень), 3, 91-97. (In Russian).

Pavlenko, M.V. and Barnov, N.G., (2019). Internal and external causes of treshinoobrazovaniya, signs of deformation of the coal seam in the area of vibration exposure. 25rd International Conference Engineering Mechanics, Czech Republic, 241-244.

Pavlenko, M.V., Barnov, N.G., Kuziev, D.A., Kenzhabayev, K.N., and Monzoyev, M.V., (2020). Vibration impact through wells and the technology of degassing of the preparation of low-permeability coal seam (Вибрационное воздействие через скважины и технология дегазационной подготовки низкопроницаемого угольного пласта). Ugol' (Журнал Уголь), 1, 36 -39. (In Russian).

Pavlenko, M.V., Guryev, S.V., Lopukhov, G.P., and Yurov, A.A., (2015). Degassing of coal seams using land-based seismic sources (Дегазация угольных пластов с использованием наземных сейсмоисточников). Proceedings of universities USMU (Известия Вузов УГГУ), 1, 42 - 46. (In Russian).

Slastunov, S.V., Yutyaev, E.P., Mazanyk, E.V., and Sadov, A.P., (2018). Development and improvement of seam degassing technologies for efficient and safe mining of coal seams (Разработка и совершенствование технологий пластовой дегазации для 
эффективной и безопасной отработки угольных пластов). Mining information and analytical bulletin (Горный информационноаналитический бюллетень), 11, 13-22. (In Russian).

Wei, J.P., Wang, H.L., Wang, D.K, and Yao, B.H, (2016). An improved model of gas flow in coal based on the effect of penetration and diffusion. Journal of China University of Mining \& Technology, 45(5), 873 - 878.
Yang, L., (2014). A mixed element method for the desorptiondiffusion-seepage model of gas flow in deformable coalbed methane reservoirs. Mathematical Problems in Engineering, 1-10.

Yao, B., Ma, Q., Wei, J., (2016). Effect of protective coal seam mining and gas extraction on gas transport in a coal seam. International Journal of Mining Science and Technology, 26(4), 637643. 